\title{
Rendimiento académico y prácticas artísticas extracurriculares en estudiantes de bachillerato
}

\author{
Academic Performance and Artistic Practice Extracurricular \\ in High School Student
}

\author{
Griselda Montoya Becerril (1) gris1831@hotmail.com \\ Roberto Oropeza Tena (1) scherzo2112@yahoo.com \\ María Luisa Ávalos Latorre (2) marilupsi@hotmail.com \\ (1) Universidad Michoacana de San Nicolás de Hidalgo \\ (2) Universidad de Guadalajara \\ (Recibido: 5 de marzo de 2017; Aceptado para su publicación: 3 de mayo de 2017)
}

Cómo citar: Montoya, G., Oropeza, R. y Ávalos M. L. (2019). Rendimiento académico y prácticas artísticas extracurriculares en estudiantes de bachillerato. Revista Electrónica de Investigación Educativa, 21, e13, 1-10. doi.10.24320/redie.2019.21.e13.1877

\section{Resumen}

El objetivo de la investigación fue analizar las diferencias entre el rendimiento académico y el tipo de práctica artística extracurricular en estudiantes de bachillerato. Participaron 169 estudiantes de la ciudad de Morelia, Michoacán (México). Se recabaron datos sociodemográficos mediante una ficha de identificación; se diseñó y aplicó un Inventario de Actividades Académicas y Extra Académicas (actividad artística entre ellas) y se obtuvo la boleta de calificaciones de cada participante; los datos se analizaron con el programa SPSS 20. Se llevaron a cabo pruebas de diferencia entre las variables: rendimiento académico y tipo de práctica artística. Se encontraron diferencias estadísticamente significativas entre el rendimiento académico y la práctica de las artes, siendo los participantes que practican actividades artísticas los de mejor rendimiento académico, sobre todo en las asignaturas de Español y Literatura.

Palabras clave: Rendimiento académico, actividad paraescolar, educación artística, bachillerato, calificaciones.

\section{Abstract}

The aim was to analyze the differences between the academic performance and the type of artistic extracurricular practice in students of high school. Participate 169 students of two high school of Morelia city, Mexico. Information was obtained socio-demographic by means of a card of identification. There was designed and applied an Inventory of Academic Activities and Extra Academic (between them, artistic activity); and there was obtained the grades of every participant. The information analyzed with the program SPSS 20.0. There were carried out tests of difference between the variables: academic performance and type of artistic practice. They found statistically significant differences between the academic performance and the practice of the arts, being the participants who practice artistic activities those of better academic performance especially in subjects of Spanish and literature. 


\section{Introducción}

El concepto de rendimiento académico surge de la necesidad de evaluar los sistemas educativos. Se entiende como el resultado del aprendizaje, promovido por la intervención pedagógica de los profesores y producido en el alumno (Montero, Villalobos y Valverde, 2007). Está relacionado con un proceso de enseñanza tomando en cuenta los factores que pueden influir en él-socioeconómicos, programas de estudio, metodología de enseñanza utilizada, la dificultad de no contar con una enseñanza personalizada y los conceptos previos (Benitez, Gimenez y Osicka, 2000, como se citó en Edel, 2003).

Existen algunos factores asociados al rendimiento académico, uno de ellos es la conducta o prerrequisitos específicos que el estudiante posee al abordar cada asignatura, las experiencias de aprendizaje, las habilidades generales para formarse o la capacidad de autodirección para el estudio. Otro aspecto importante es el grado de implicación en el proceso, el cual depende fundamentalmente de su motivación, que ayuda a la persona a enfrentar los factores ambientales: socioeconómicos, culturales, familiares, afectivos y de personalidad (Forero, 1991, como se citó en Mujica, Thomas y Mennella, 2009).

El rendimiento académico es un término complejo. Pese a que se debe entender a partir de sus procesos de evaluación, no es suficiente. De acuerdo con Edel (2003), es necesario conocer otros elementos que influyen, tales como el grupo de pares, el aula o el contexto educativo y otros aspectos e intereses personales, de ahí la importancia de estudiarlo en relación con otras variables.

En un intento por definir este término retomamos a Maquilón y Hernández (2012), quienes lo conceptualizan como el conjunto de habilidades, destrezas, hábitos, ideales, aspiraciones, intereses, inquietudes y realizaciones que aplica el estudiante para aprender. Por otro lado, Edel (2003) considera que el indicador del nivel de aprendizaje que ha alcanzado el estudiante, así como el resultado del esfuerzo y la capacidad de trabajo que ha tenido durante el proceso de enseñanza-aprendizaje, se puede sintetizar en una estimación numérica. A partir de esta valoración es posible identificar el nivel de conocimientos de un estudiante en una materia o en determinada área, en relación con la edad y el nivel académico.

El rendimiento académico es un indicador importante de la calidad educativa, ya que es un predictor de éxito o fracaso escolar, y debe ser analizado a la par de las diversas variables que influyen en los procesos de aprendizaje, por ello es considerado un problema de relevancia dentro del sistema educativo y de todos sus actores que trasciende el contexto escolar.

En México el bachillerato se cursa durante la adolescencia (entre los 15 y los 18 años, aproximadamente), etapa considerada como proceso biopsicosocial de transición de la vida, en ese período las personas atraviesan cambios tales como la búsqueda de identidad, la independencia de los padres, así como la aceptación de sus pares en diferentes círculos sociales (Gaxiola, González, Contreras y Gaxiola, 2012).

Desde el punto de vista educativo, al cursar el bachillerato este grupo etario presenta como problema principal la deserción escolar o abandono del sistema educativo (Hernández, Álvarez y Aranda, 2017), debido principalmente a un bajo rendimiento escolar, un inadecuado desarrollo, problemas familiares y de comunicación interpersonal, entre otros. Esta problemática no es exclusiva de México, las estadísticas a nivel internacional reportan que, por ejemplo, en España el $42.3 \%$ de los alumnos acumula retrasos antes de cursar el nivel medio superior, lo que aumenta la probabilidad de fracaso escolar (Calero, Choi y Waisgrais, 2010).

El Informe de Educación Media Superior en México (Instituto Nacional de Evaluación de la Educación [INEE], 2011) realizó una revisión del comportamiento de las tasas de deserción, que pasó de 19.8\% a 14.9\% en 13 años. Por su parte, la Secretaría de Educación Pública (SEP, 2014), afirmó que el 13.1\% de los estudiantes de educación media superior (ciclo 2013-2014) tuvo un mayor fracaso escolar, lo que se reflejó en el nivel de deserción.

El rendimiento académico tiene una gran importancia en ámbitos educativos, por lo que ha sido objeto de interés para investigadores de distintas disciplinas. A partir de una revisión de los estudios realizados en 
los últimos años en torno al tema, destacan investigaciones sobre la importancia de la familia y el grado de estudios de los padres (Chaparro, González y Caso, 2016; Hayakawa, Velazco, Sifuentes y Landeros, 2013); la influencia del turno escolar (Vera, Huesca y Laborín, 2012); las diferencias entre géneros (Caso y Hernández 2007; Stover, Uriel, Iglesia, Freiberg y Liporace, 2014; Rodríguez, Fita y Torrado, 2003); la actividad laboral (Carrillo y Ríos, 2013); las estrategias de aprendizaje (Roux y Anzures, 2015) así como las estrategias de enseñanza (García, Urionabarrenetxea y Bañales, 2016) y las habilidades de estudio, tales como una mayor organización, planificación (Capdevila y Bellmunt, 2016) y motivación (Becerra y Reidl, 2015; Stover et al., 2014).

Además de los estudios antes referidos, en donde el énfasis radica en elementos educativos y sociodemográficos, hay investigaciones donde algunos aspectos psicológicos han sido de interés para explicar el rendimiento académico. Resaltan aquellos sobre la autoeficacia percibida para el estudio (Cartagena, 2008; González, 2010; Hernández y Barraza, 2014; Serra, 2010), los que en general concluyen que los estudiantes con una percepción de autoeficacia alta tienden a ser más autodidactas y a enfrentar con mayor esfuerzo los obstáculos escolares que se les presentan. Otros estudios se han interesado en identificar variables psicológicas que pudieran explicar el éxito escolar, por ejemplo, los rasgos de personalidad (Cupani y Zalazar, 2014), el burnout (Portolés y González, 2015) y la resiliencia (Gaxiola et al., 2012; Gaxiola, González y Gaxiola, 2013).

\subsection{Importancia de la actividad artística}

Pese que a nivel social el arte sufre desprestigio y sigue siendo una actividad concebida para diletantes de la cual, se considera, podríamos prescindir (Palacios, 2006), en la actualidad la práctica artística ha cobrado auge como actividad recreativa u ornamental, sobre todo en la población infantil y adolescente, sin tomar en cuenta que su valor cultural y personal es significativo.

A través de los años la educación se ha propuesto ofrecer una formación integral del individuo; en este sentido, la práctica artística ha cobrado importancia al ser fuente potencializadora de habilidades de pensamiento y de expresión individual, ello a partir del proceso sistemático y riguroso que implica su ejercicio. La práctica de una actividad artística, ejercita el pensamiento reflexivo y analítico, su enseñanza requiere fundamentación técnica, es un proceso complejo (Garaigordobil y Pérez, 2002), además de la adquisición de las habilidades se considera que la práctica de alguna actividad artística favorece el desarrollo de otros repertorios académicos, incluso se ha afirmado que se transfieren las habilidades artísticas a otras áreas del conocimiento (Winner, Goldstein y Lancrin, 2014).

Winner et al. (2014) llevaron a cabo un meta-análisis en el año 2000, en el que el interés central fue identificar las habilidades desarrolladas mediante la educación artística, y encontraron que el nivel de logro escolar de los alumnos que tomaban cursos de arte era mayor al que los que no los tomaban. Aunque a la fecha hay poca evidencia al respecto, destaca el estudio realizado por Parres y Flores (2011), quienes determinaron el efecto de los cursos de artes visuales en el nivel universitario sobre la motivación y las estrategias de autorregulación para el aprendizaje. La comparación de los puntajes escolares antes y después del curso mostró un efecto positivo en la motivación, pero no en las estrategias de aprendizaje, observando también un efecto en la valoración de sus capacidades para aprender.

Otro estudio hecho con población infantil realizado por Krumm y Lemos (2012) evaluó en 301 niños de 8 a 14 años de edad, sus actividades artísticas y sus habilidades creativas, para lo cual se realizó una evaluación multicomponente en la que se utilizaron técnicas como los sociogramas y las escalas, así como diferentes informantes; los autores encontraron que los niños que realizaban actividades artísticas obtuvieron puntuaciones más elevadas en la creatividad -desde el punto de vista de los padres y del propio niño. Por su parte, Ponzón (2014) indagó sobre la importancia del involucramiento estudiantil del universitario en actividades extracurriculares y su repercusión en el desempeño académico; mediante grupos focales (con 75 personas) el autor analizó la interacción discursiva y la contrastación de las opiniones de sus miembros. Todos los grupos consideraron a las actividades extracurriculares como un elemento importante en el contexto universitario, sobre todo porque -consideraron-fortalece las relaciones entre los estudiantes y diversifica su formación. 
Una última investigación encontrada en población universitaria es la de Ferreyra, Oropeza y Ávalos (2015), quienes analizaron la relación de involucramiento y motivación artística con el rendimiento académico, encontrando correlaciones significativas entre involucramiento, motivación artística y rendimiento académico, así como entre involucramiento, motivación artística y el promedio de calificaciones de algunas asignaturas. Se encontró un efecto ligeramente adverso entre la práctica de las artes y el rendimiento académico.

Tal como podemos apreciar, la poca evidencia que existe al respecto no descarta el hecho de que la práctica de actividades artísticas repercute en el rendimiento académico. En este sentido, Touriñán (2011) afirma que la educación artística es una forma de educación en valores que está implicada pedagógicamente en los problemas de conocer, estimar, enseñar, elegir, realizar y sentir los valores estéticos y artísticos; por su parte, López, San Pedro y González (2013) consideran que la práctica del arte está delimitada por las restricciones naturales de los contextos de la persona y sus propias normas, y afirman que las motivaciones pueden ser múltiples: canalización del impulso creativo, fines terapéuticos, motivos económicos, mejora de la técnica, entre otras.

Ante estos hallazgos y la poca evidencia al respecto, el objetivo de esta investigación es analizar la relación entre rendimiento académico y el tipo de práctica artística extracurricular en estudiantes de bachillerato.

\section{Método}

En el estudio participaron voluntariamente 169 estudiantes de sexto semestre de bachillerato de la ciudad de Morelia, Michoacán. La muestra fue no probabilística por conveniencia; 48 \% fueron varones y $52 \%$ fueron mujeres, con un promedio de edad de 17.8 años $(D E=.81)$. Todos los estudiantes firmaron una carta de consentimiento informado en la que los jóvenes aceptaban participar y que sus datos fueran publicados.

Como instrumentos se emplearon una ficha de identificación, en la que se solicitó información sociodemográfica de los estudiantes y un inventario sobre actividades académicas y extra-académicas (diseñado específicamente para esta investigación) que incluía 55 reactivos con respuestas de opción múltiple. El inventario (de autoaplicación) evalúa cuatro aspectos: 1) actividades cotidianas, clima escolar, logros académicos e interés académico; 2) motivación e involucramiento personal y familiar en las artes; 3) motivación e involucramiento personal y familiar en la práctica deportiva; y 4) motivación e involucramiento personal y familiar en algún servicio comunitario. Cabe señalar, que para fines de este escrito se analizaron los datos correspondientes al punto 2, el cual se centra en las prácticas artísticas extracurriculares (Ferreyra, Oropeza y Avalos, 2015).

Para abordar la práctica artística se consideró la motivación e involucramiento, la primera como una valoración subjetiva de los participantes en una escala de baja a alta, mientras que para el involucramiento se consideró la edad de inicio, los años realizándola, el número de días y las horas semanales dedicadas a la actividad.

Por último, se obtuvo la boleta de calificaciones escolares -listado de materias cursadas hasta el momento de la recolección de datos y las calificaciones correspondientes en una escala de 0 a 10. La boleta se solicitó a los participantes, quienes la imprimieron desde el servidor escolar de la universidad a la que estaban adscritos.

En el estudio, analítico-descriptivo de evaluación única con metodología cuantitativa, se usó una prueba $t$ рага grupos independientes para analizar las diferencias entre las variables consideradas en la investigación. Con la autorización previa del profesor, la recolección de la información se llevó a cabo en los salones de clase de dos bachilleratos de la ciudad de Morelia. Se informó a los participantes los objetivos de la investigación y se solicitó su colaboración. Los estudiantes de sexto semestre que aceptaron participar firmaron un consentimiento informado en el que autorizaron el uso de sus datos para fines de divulgación científica. Las indicaciones pertinentes sobre el llenado de los instrumentos se dieron de manera grupal. Todas las dudas planteadas fueron resueltas en el momento de la aplicación. 
Para contestar el inventario los participantes emplearon, en promedio, 90 minutos. El inventario fue administrado en grupos de aproximadamente 40 estudiantes, ya que cada clase tenía, en promedio, ese número de alumnos. Al finalizar el inventario se les solicitó que descargaran del sistema escolar su boleta con las calificaciones registradas hasta el momento.

\section{Resultados}

Respecto a las calificaciones que dan cuenta del rendimiento académico, se observó que el 79\% de los estudiantes reprobó al menos una materia durante el bachillerato, obteniendo un promedio de calificación global de 7.92 ( $\mathrm{DE}=.766$ ); además de este dato se obtuvieron las calificaciones de las asignaturas de Español y Matemáticas cursadas durante el bachillerato.

El 37\% de los participantes dijo practicar algún tipo de actividad artística fuera de las actividades académicas. Entre las actividades artísticas realizadas destaca: música con el $21 \%$ de los participantes, danza o ballet con el $12.4 \%$ y literatura con el $10.7 \%$, el resto se distribuyó en fotografía, pintura, teatro, escultura y cine.

Al realizar las comparaciones entre el promedio de calificaciones y el tipo de práctica extracurricular se obtuvo lo siguiente: el promedio de calificaciones más alto se encontró en los participantes que practican música. Se registraron diferencias estadísticamente significativas entre la práctica de música y el rendimiento académico $(t=-2,17, g l=33, p=.037)$, así como entre la práctica de música y la calificación en la asignatura de Literatura III ( $t=2.39, \mathrm{gl}=177, \mathrm{p}=.018)$, Literatura IV $(\mathrm{t}=-2.666, \mathrm{gl}=70.488, \mathrm{p}=0.01)$ y Taller de Lectura II ( $t=-2.428, g l=21.475, p=0.024)$.

Por otro lado, en cinco asignaturas y el promedio global fue más alto en los estudiantes que practican escultura $(M=9.33)$ respecto a los que no practican $(M=8.33)$. En este sentido, se observan diferencias significativas con la asignatura de Literatura IV $(t=4.33, g l=7.04, p=0.031)$.

Respecto a la práctica de Literatura como actividad artística se observaron diferencias estadísticamente significativas con el promedio de calificaciones global entre los que sí practican $(M=8.46)$ y los que no $(M=7.86)(t=-3.340, g l=167, p=0.001)$. Además, se encontraron diferencias estadísticamente significativas con las asignaturas de Taller de Lectura y Redacción I y II, y Literatura II y IV (ver tabla I).

Tabla I. Diferencias entre la práctica de Literatura y las calificaciones en asignaturas

\begin{tabular}{l|c|c}
\hline \multicolumn{1}{c|}{ Asignatura } & Promedio escolar & Prueba de diferencias \\
\hline Taller de Lectura y Redacción I & Sí: $M=8.83$ & $t=-2.103, g l=167, p=0.037$ \\
& No: $M=8.14$ & \\
Taller de Lectura y Redacción II & Sí: $M=9.28$ & $t=-3.288, g l=167, p=0.001$ \\
& No: $M=8.27$ & \\
Literatura II & Sí: $M=9.06$ & $t=-2.756, g l=167, p=0.007$ \\
& No: $M=8.14$ & \\
Literatura IV & Sí: $M=9.06$ & $t=2.352, g l=167, p=0.012$ \\
& No: $M=8.28$ & \\
\hline
\end{tabular}

Sí= Estudiantes que sí practican Literatura.

$\mathrm{No}=$ Estudiantes que no practican Literatura.

No se observaron diferencias entre el promedio de calificaciones por asignatura y el promedio global y la práctica de cine. En la actividad artística de fotografía se observan diferencias estadísticamente significativas con el promedio global de calificaciones ( $t=2.555, g l=167, p=0.014)$, así como con las asignaturas de Literatura III e Inglés V (ver tabla II). 
Tabla II. Diferencias entre la práctica de Fotografía y las calificaciones en asignaturas

\begin{tabular}{l|c|c}
\hline \multicolumn{1}{c|}{ Asignatura } & Promedio escolar & Prueba de diferencias \\
\hline Literatura III & Sí: $M=8.98$ & $t=-2.690, g l=20.599, p=0.014$ \\
& No: $M=8.16$ & \\
Inglés V & Sí: $M=8.69$ & $t=-2.092, g l=167, p=0.038$ \\
& No: $M=7.99$ & \\
\hline
\end{tabular}

Sí= Estudiantes que sí practican Fotografía.

No= Estudiantes que no practican Fotografía.

En la práctica de teatro se observó que el promedio de calificaciones en todas las asignaturas es más alto en aquellos que sí lo practican, exceptuando la asignatura de Matemáticas. Por otro lado, existen diferencias estadísticamente significativas entre la práctica de esta actividad y el promedio global de calificaciones $(t=2.798, g l=167, p=0.006)$ y en las asignaturas de Taller de Lectura y Redacción II y Literatura III y IV (ver tabla III).

Tabla III. Diferencias entre la práctica de Teatro y las calificaciones en asignaturas

\begin{tabular}{l|c|c}
\hline \multicolumn{1}{c|}{ Asignatura } & Promedio escolar & Prueba de diferencias \\
\hline Taller de Lectura y Redacción II & Sí: $M=9.17$ & $t=2.271, g l=167, p=0.024$ \\
& No: $M=8.16$ & \\
Literatura III & Sí: $M=9.25$ & $t=4.482, g l=17.538, p=0.0003$ \\
& No: $M=8.16$ & \\
Literatura IV & Sí: $M=9.25$ & $t=3.994, g l=16.123, p=0.001$ \\
& No: $M=8.29$ & \\
\hline
\end{tabular}

Sí= Estudiantes que sí practican Teatro. No= Estudiantes que no practican Teatro.

Por último, se observaron algunas diferencias entre la práctica de danza/ballet y el promedio global de calificaciones ( $t=2.517, \mathrm{~g}=167, \mathrm{p}=0.013)$ entre los que sí practican $(M=8.31)$ y los que no $(M=7.87)$, así como el promedio de calificaciones en Matemáticas I, II y IV; Literatura III y IV e Inglés V (ver tabla IV).

Tabla IV. Diferencias entre la práctica de Danza/Ballet y las calificaciones en asignaturas

\begin{tabular}{l|c|c}
\hline \multicolumn{1}{c|}{ Asignatura } & Promedio escolar & Prueba de diferencias \\
\hline Matemáticas I & $\begin{array}{c}\text { Sí: } M=7.9 \\
\text { No: } M=7.17\end{array}$ & $t=2.684, g l=29.736, p=0.06$ \\
Matemáticas III & $\begin{array}{l}\text { Sí: } M=8.05 \\
\text { No: } M=7.26\end{array}$ & $t=3.050, g l=30.073, p=0.005$ \\
& $\begin{array}{l}\text { Sí: } M=8.76 \\
\text { No: } M=8.16\end{array}$ & $t=1.907, g l=167, p=0.058$ \\
Literatura III & Sí: $M=8.86$ & $t=1.958, g l=167, p=0.052$ \\
Literatura IV & No: $M=8.29$ & \\
Satemáticas IV $M=8.24$ & $t=2.471, g l=167, p=0.014$ \\
& No: $M=7.35$ & \\
Inglés V & Sí: $M=8.95$ & $t=3.535, g l=167, p=0.001$, \\
& No: $M=7.93$ & \\
\hline
\end{tabular}

Sí= Estudiantes que sí practican Danza/Ballet. $\mathrm{No}=$ Estudiantes que no practican Danza/Ballet.

\section{Discusión y conclusiones}

Como evidencian los resultados, parece que la práctica de actividades artísticas sí favorece el desempeño académico escolar, y entre las diferentes disciplinas artísticas, quienes practican música son los que mejores calificaciones obtuvieron en el bachillerato; sin embargo, habría que tomar estos resultados con precaución debido al tamaño de los grupos analizados en algunas de las prácticas analizadas. 
Destacan las asignaturas relacionadas con la expresión escrita y la literatura como las más favorecidas para la práctica de actividades artísticas, tal parece que esas asignaturas proporcionan habilidades que favorecen la experiencia de aprendizaje (Forero, 1991, como se citó en Mujica, Thomas y Mennella, 2009). A su vez, esto puede ser una fuente de alta motivación, ya que los estudiantes pueden sentirse más seguros al percibir con mayor sencillez las actividades derivadas de las mismas, tal como lo refieren investigaciones referidas en párrafos anteriores (Cartagena, 2008; Caso y Hernández, 2007; González, 2010, entre otros).

Aunque a la fecha existen pocos estudios similares al aquí realizado, detectamos coincidencias con nuestros hallazgos: Parres y Flores (2011) concuerdan en que la práctica artística impacta positivamente en la motivación y las estrategias de autorregulación para el aprendizaje; en ese mismo sentido, Ferreyra, Oropeza y Ávalos (2015) también encuentran beneficios de la práctica artística en el rendimiento académico en estudiantes universitarios. Por su parte, Krumm y Lemos (2012) identificaron un mayor rendimiento académico y mayor creatividad en niños de primaria que estudiaban alguna disciplina artística, mientras que Ponzón (2014) afirma que la práctica de actividades artísticas extracurriculares favorece la formación integral de los jóvenes.

Los hallazgos de este y otros estudios nos lleva a suponer que la práctica de alguna disciplina artística favorece positivamente el rendimiento académico en el promedio general de calificaciones, así como en algunas asignaturas específicas en cualquier nivel educativo. Por ello resulta fundamental inculcar estas prácticas desde edades tempranas y mantenerlas hasta la vida adulta. Al respecto, Winner, Goldstein y Lancrin (2014) afirman que la educación artística ayuda a desarrollar habilidades que mejoran el desempeño de los alumnos en las materias académicas no artísticas, como las matemáticas, las ciencias, la lectura y la escritura, así como a fortalecer la motivación académica, la confianza en sí mismo y la capacidad de comunicarse y cooperar de manera eficaz.

En la muestra estudiada destaca el hecho de que sólo una tercera parte de los estudiantes encuestados practica alguna actividad artística; por lo que es indispensable promover estas actividades poco difundidas en la población general en México.

Posteriores investigaciones deben buscar entender cómo es que las habilidades adquiridas o desarrolladas en algunas actividades artísticas son transferidas a la enseñanza formal. Winner, Goldstein y Lancrin (2014), por ejemplo, afirman que el aprendizaje musical implica el entrenamiento auditivo, de modo que dicho aprendizaje se extiende a las habilidades de la percepción del habla; el aprendizaje musical se parece al aprendizaje escolar, pues involucra la disciplina, la práctica y la lectura de notas, y esto se extiende al dominio del desempeño académico.

Cerquera (2014), afirma que, a pesar de existir muchas investigaciones sobre rendimiento académico, aún no se ha llegado a un consenso sobre cómo determinar los factores del rendimiento académico, ya que en la realidad son muchas las características del individuo, la escuela o el sistema que se relacionan entre sí de diferente manera y pueden afectar el logro estudiantil. La presente investigación ofrece un acercamiento a aquellas variables extracurriculares que pudieran estar relacionadas con el rendimiento académico, así como su relación específica con algunos de los contenidos formales. Pese a este acercamiento, resulta indispensable seguir profundizando al respecto.

\section{Referencias}

Becerra, C. y Reidl, L. (2015). Motivación, autoeficacia, estilo atribucional y rendimiento escolar de estudiantes de bachillerato. Revista Electrónica de Investigación Educativa, 17(3), 79-93. Recuperado de https://redie.uabc.mx/redie/article/view/664/1297

Calero, J., Choi, A. y Waisgrais, S. (2010). Determinantes de riesgo de fracaso escolar en España: una aproximación a través de un análisis logístico multinivel aplciado a PISA-2006. Revista de Educación, Supl., 225-256. Recuperado de http://www.revistaeducacion.educacion.es/re2010/re2010 09.pdf 
Capdevila, A. y Bellmunt, H. (2016). Importancia del os hábitos de estudio en el rendimiento académico del adolescente: diferencias por género. Education Siglo XXI, 34(1), 157-172. Recuperado de https://www.researchgate.net/publication/313864438 Importancia de los habitos de estudio en el r endimiento academico del adolescente diferencias por genero

Carrillo, S. y Ríos, J. (2013). Trabajo y rendimiento escolar de los estudiantes universitarios. El caso de la Universidad de Guadalajara, México. Revista de la Educación Superior, 42(166), 9-34. Recuperado de http://publicaciones.anuies.mx/pdfs/revista/Revista166 S1A1ES.pdf

Cartagena, M. (2008). Relación entre la autoeficacia y el rendimiento escolar y los hábitos de estudio en alumnos de secundaria. Revista Iberoamericana sobre Calidad, Eficacia y Cambio en Educación, 6(3), 60-99. Recuperado de http://www.rinace.net/arts/vol6num3/art3.pdf

Caso, J. y Hernández, L. (2007). Variables que inciden en el rendimiento académico de adolescentes mexicanos. Revista Latinoamericana de Psicología, 39(3), 487-501.

Cerquera, O. (2014). Estado del arte del rendimiento académico en la educación media. Rhec, 17(17), 197220. Recuperado de http://revistas.udenar.edu.co/index.php/rhec/article/view/2091/pdf 11

Chaparro, A., González, C. y Caso, J. (2016). Familia y rendimiento académico: configuración de perfiles estudiantiles en secundaria. Revista Electrónica de Investigación Educativa, 18(1), 53-68. Recuperado de http://redie.uabc.mx/redie/article/view/774

Cupani, M. y Zalazar, M. (2014). Rasgos complejos y rendimiento académico: contribución de los rasgos de personalidad. Revista Colombiana de Psicología, 23(1), 57-71. doi:10.15446/rcp.v23n1.39774

Edel, R. (2003). El rendimiento académico: concepto, investigación y desarrollo. Revista Electrónica Iberoamericana sobre Calidad, Eficacia y Cambio en Educación, 1(2), Recuperado de https://revistas.uam.es/index.php/reice/article/view/5354

Ferreyra, D. A., Oropeza, R. y Ávalos M. L. (2015). Relación entre la práctica de las artes y el rendimiento académico en estudiantes universitarios. Sinéctica, Revista Electrónica de Educación, (44), 1-15.

Recuperado de https://sinectica.iteso.mx/index.php/SINECTICA/article/view/162

Garaigordobil, M. y Pérez, J. (2002). Efectos de la participación en el programa de arte Ikertze sobre la creatividad verbal y gráfica. Anales de Psicología, 18(1), 95-110. Recuperado de

https://www.redalyc.org/articulo.oa?id=16718106

García, J. D., Urionabarrenetxea, S. y Bañales, A. (2016). Cambios en metodologías docente y de evaluación: ¿mejoran el rendimiento del alumnado universitario? Revista Electrónica de Investigación Educativa, 18(3), 1-18. Recuperado de http://redie.uabc.mx/redie/article/view/691

Gaxiola, J., González, S., Contreras, Z. y Gaxiola, E. (2012). Predictores del rendimiento académico en adolescentes con disposiciones resilientes y no resilientes. Revista de Psicología, 30(1), 49-74. Recuperado de http://revistas.pucp.edu.pe/index.php/psicologia/article/view/2629/2576.

Gaxiola, J., González, S. y Gaxiola, E. (2013). Autorregulación, resiliencia y metas educativas: variables protectoras del rendimiento académico de bachilleres. Revista Colombiana de Psicología, 22(2), 241-252. Recuperado de http://www.scielo.org.co/pdf/rcps/v22n2/v22n2a02.pdf

González, M. (2010). Autoeficacia percibida y desempeño académico en estudiantes universitarios. Memorias del Congreso Iberoamericano de Educación. Argentina: Universidad Nacional de Cuyo. Recuperado de http://www.adeepra.org.ar/congresos/Congreso\%20IBEROAMERICANO/ACCESO/RLE2870 Gonzalez.pdf 
Hayakawa, A., Velazco, P., Sifuentes, E. y Landeros, B. (2013). Análisis del efecto de la preparatoria en el rendimiento académico de los estudiantes de una institución de educación superior. Revista Internacional de la Educación en Ingeniería, 6(1), 14-22. Recuperado de http://www.academiajournals.com/revista-ed-ening/

Hernández, P., Álvarez, M. y Aranda, A. (2017). El problema de deserción escolar en la produccción científica educativa. Revista Internacional de Ciencias Sociales y Humanidades, 1, 89-112. Recuperado de https://www.redalyc.org/pdf/654/65456040007.pdf

Hernández. L. y Barraza, A. (2014). Autoeficacia académica percibida en la educación superior. Revista Electrónica de la Red Durango de Investigadores Educativos, 6(10), 67-77. Recuperado de https://dialnet.unirioja.es/servlet/articulo?codigo=6553281.

Instituto Nacional para la Evaluación de la Educación. (2011). La educación media superior en México, informe 2010-2011. INEE: México. Recuperado de

http://www.inee.edu.mx/images/informe2011/informe2011final.pdf

Krumm, G. y Lemos, V. (2012). Actividades artísticas y creatividad en niños escolarizados argentinos. International Journal Psychological Research, 5(2), 40-48. Recuperado de http://www.scielo.org.co/pdf/ijpr/v5n2/v5n2a05.pdf

López, I., San Pedro, J. y González, C. (2013). La motivación en el área de expresión plástica. Arte, Individuo y Sociedad, 26(2), 199-213. Recuperado de http://revistas.ucm.es/index.php/ARIS/article/view/41265

Montero, E., Villalobos, J. y Valverde, A. (2007). Factores institucionales, pedagógicos, psicosociales y sociodemográficos asociados al rendimiento académico en la Universidad de Costa Rica: Un análisis multinivel. RELIEVE, 13(2), 215-234. Recuperado de www.uv.es/RELIEVE/v13n2/RELIEVEv13n2 5.html

Maquilón, S. y Hernández, P. (2012). Influencia de la motivación en el rendimiento académico de los estudiantes de formación profesional. Revista Electrónica Interuniversitaria de Formación del Profesorado, 14(1), 81-100. Recuperado de https://www.redalyc.org/articulo.oa?id=217017192007

Mujica, A., Thomas, I. y Mennella, M. (2009). Relación entre la motivación y el rendimiento en estudiantes de lenguas modernas. Revista Omnia, 15(1), 143-161. Recuperado de

http://132.248.9.34/hevila/OmniaMaracaibo/2009/vol15/no1/9.pdf

Palacios, L. (2006). El valor del arte en el proceso educativo. Reencuentro, 46. Recuperado de

http://bidi.xoc.uam.mx/resumen articulo.php

Parres, S. y Flores, R. (2011). Experiencia educativa en arte visual diseñada bajo un modelo de autorregulación del aprendizaje con estudiantes universitarios. Revista Mexicana de Investigación Educativa, 16(49), 597-624. Recuperado de

http://www.comie.org.mx/revista/v2018/rmie/index.php/nrmie/article/view/385

Ponzón, J. (2014). Los estudiantes universitarios ante las actividades extracurriculares. Revista Andaluza de Ciencias Sociales, 13, 137-150. doi:10.12795/anduli.2014.i13.08

Portolés, A. y González, J. (2015). Rendimiento académico y correspondencias con indicadores de salud física y psicológica. Revista Técnico-Científica del Deporte Escolar, Educación Física y Psicomotricidad, 1(2), 164-181. Recuperado de https://core.ac.uk/download/pdf/75988294.pdf

Rodríguez, S., Fita, E. y Torrado, M. (2003). El rendimiento académico en la transición secundariauniversidad. Revista de Educación, 334, 391-414. Recuperado de

http://www.revistaeducacion.educacion.es/re334/re334 22.pdf 
Roux, R. y Anzures, G. (2015). Estrategias de aprendizaje y su relación con el rendimiento académico en estudiantes de una escuela privada de Educación Media Superior. Revista Electrónica Actualidades Investigativas en Educación, 15(1), 1-16. Recuperado de https://www.redalyc.org/articulo.oa?id=44733027014

Secretaría de Educación Pública. (2014). Principales cifras del Sistema Educativo Nacional 2013-2014. Recuperado de https://www.planeacion.sep.gob.mx/Doc/estadistica e indicadores/principales cifras/principales cifras 20132014 bolsillo.pdf

Serra, J. (2010). Autoeficacia y rendimiento académico en estudiantes universitarios. Revista Griot, 3(2), 37-45. Recuperado de https://revistas.upr.edu/index.php/griot/article/view/1877

Stover, J., Uriel, F., Iglesia, G., Freiberg, A. y Liporace, M. (2014). Rendimiento académico, estrategias de aprendizaje y motivación en alumnos de Escuela Media de Buenos Aires. Perspectivas en Psicología, 11(2), 10-20. Recuperado de https://dialnet.unirioja.es/servlet/articulo?codigo=5113960

Touriñán, J. (2011). Claves para aproximarse a la educación artística en el sistema educativo: educación "por" las artes y educación "para" un arte. Estudios sobre educación, 21, 61-81. Recuperado de http://hdl.handle.net/10171/22549

Vera, J., Huesca, L. y Laborín, J. (2012). Logro y tasa de riesgo en alumnos de alto y bajo desempeño escolar en el nivel medio superior en Sonora. Perfiles Educativos, 33(132), 48-66. Recuperado de https://www.redalyc.org/html/132/13218510004/

Winner, E., Goldstein, T. y Lancrin, S. (2014). ¿El arte por el arte? La influencia de la educación artística. México: OECD. doi 10.1787/9789264224902-es 